\title{
Advanced Computer Communication and Control Technology for Modern Substations
}

\author{
Swetha $\mathbf{K}^{1}$, Rashmi H $\mathbf{C}^{2}$ \\ Assistant Professor, Department of TCE, GSSSIETW, Mysuru, India ${ }^{1}$ \\ Assistant Professor, Department of ECE, GSSSIETW, Mysuru, India ${ }^{2}$
}

\begin{abstract}
This paper proposes a novel approach to achieve monitoring and control of modern substations using advanced computer communication and control technology. The proposed approach is based on a code division multiple access (CDMA) and interleave division multiple access (IDMA) system joining with optical-fiber communication technology to realize the utility infrastructure of Smart Grid. Recently, owing to the rapid growth on optical-fiber communication, it can offer standardized communication technology for wide-area, metropolitan-area, and local-area networks. Moreover, optical-fiber communication technologies offer not only significant benefits on connection among different CDMA-IDMA systems such as rapid deployment, higher baud rate (equal or higher than 14 $\mathrm{Tb} / \mathrm{s}$ ), enhanced bandwidth, less bit error rate (equal or less than $10^{-9}$ ), less optical-amplifier quantities, reduced interference among them, lower signal attenuation, etc. but also the advantages of being more suitable for remote-end applications in Smart Grid environment. With spreading spectrum modulation and demodulation combing encoding, it can be used to transmit data and images over power lines to accomplish opportunities and challenges in the effort on comparisons and assure safety, security, and reliability in order to achieve reliability and demand efficiency for modern substations of the utility system as a supervisory control and data acquisition (SCADA) system.
\end{abstract}

Keywords: Code division multiple access, interleave division multiple access, optical-fiber communication, supervisory control and data acquisition.

\section{INTRODUCTION}

In recent years, there have been endeavors to modernize the power grid, variously known as 'Smart Grid', 'IntelliGrid', 'GridWise', 'Modern Grid', etc. The Smart Grid of modernization electricity delivery system brings challenges in the effort to assure safety, security, and reliability for utilities and consumers. The delivery circuit of Smart Grid is a form of electrical network to achieve the main functions such as monitoring, protection, etc. Through distribution substation systems and high-voltage transmission networks, the delivery circuit distributed generators, to industrial users and building automatically optimizes the operation of the interconnected elements from the control central and automation substation systems, to distributed energy storage installations, and finally to end-use consumers.

Smart Grid can be simply divided into power part and information part. The power part is related to the electrical networks while the information part (e.g., data, voice, video, etc.) is with the communication networks. According to International Telecommunications Union (ITU), Smart Grid has been applied to standards and protocol of optical communication. Power-line communication (PLC) and optical-fiber communication technologies are suitable to Smart Grid. Advanced optical communication system offer the benefits of inexpensive products, rapid deployment, high capacity, enhancement bandwidths, wavelength division multiplexing, and connected mobile communications which exhibit better efficiency, wider bandwidth, and less bit error rate (BER) than wireless technologies.

Some studied systems present various smart applications using standardized optical communication technologies, e.g., IEEE 802.3ah based Ethernet Optical Network, ITUT G.987 based Broadband Optical Network, ITU-T G.984.1 ATM Optical Network, Optical Network of passive and active based electrical signal/optical signal conversion, etc.

The system with optical communication and PLC operated as a supervisory control and data acquisition (SCADA) system is used to deliver electricity from different points of generation to various consumers. The transmission-grid revolution is necessary to recognize and deal with more diversified challenges than in advance.

This paper proposes a new approach based on interleave division multiple access and code division multiple access (IDMA/CDMA) as well as optical IDMA and optical CDMA (OIDMA/OCDMA) hybrid transmission architecture to achieve monitoring and control of modern substations. The OIDMA/OCDMA technique is used as the uplink transmission while the CDMA/OCDMA technique is used as the downlink transmission for monitoring and control of electric power systems. 


\section{INTEGRATION OF SCADA SYSTEMS AND SMART GRID}

\section{A. SCADA Systems}

A supervisory control and data acquisition (SCADA) system composes of human-machine interface (HMI), supervisory system, remote terminal units (RTU), Programmable logic controllers (PLCs), communication Infrastructure, and SCADA programming, that provides security, protection, monitor, distribution, and analysis of real- time environmental data from a simple office building to a complex nuclear power plant [3].

The HMI system is an input-output device that presents the process data to be controlled by a human operator that offer the operating personnel to see the information graphically. The supervisory system is employed as a server for communicating between the equipment of the SCADA system such as RTUs, PLCs and sensors, etc., and the HMI software used in the control room workstations. The communication infrastructure including power line/optical fiber combining wireless connections is used for the SCADA system. The SCADA programming in a master or an HMI is used for creating maps and diagrams which offer important situational information in case of an event failure or a process failure. The process control systems (PCSs) are connected to the sensors for collecting the sensor output signals in order to convert the sensor signals into digital data, that are designed to automate electronic systems based on a predetermined set of conditions, such as traffic control or power grid management. The RTUs are used to transmit telemetry data to the supervisory system and receive the messages from the master system for controlling the connected objects. Some PLCs are inclusive of one or more RTUs and or connected to any number of actuators and sensors in order to gather and analyse the real-time data to keep track, monitor, and control industrial equipment in different types of industries.

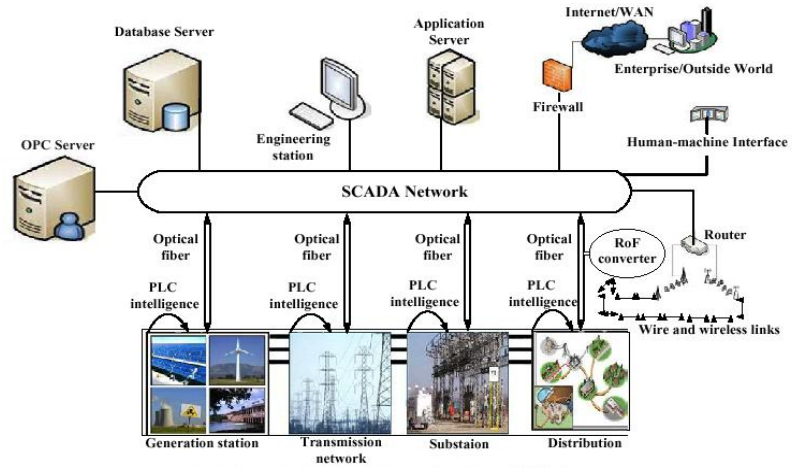

Fig. 1.An overview of the high-level construction of the studied SCADA system

Fig. 1 shows an overview of the high-level construction of the studied SCADA system, where the remote station may be the studied modern power substation. The SCADA system network may consist of power-line communication, optical- fiber communication, wireless segments, etc. with other organization network On different network segments. With advanced information analysis Obtained from different Instruments in the computing field, the integration of digital electronics devices plays an important role in the manufacturing industry. Using Power line/optical-fiber connections, the manufacturing plants employ PLCs/RTUs to control the devices and develop distributed and large complicated systems in which smart systems are parts of the processes of the manufacturing monitoring and control systems. The SCADA systems can monitor and execute extra regular values changes to optimum functions and these systems are considered two-way loop systems and run with relatively little human intervention. One of the key processes of the SCADA systems is the ability to monitor an entire system in real time. This is facilitated by data acquisitions including meter reading, checking statuses of sensors, etc. that are communicated at regular values depending on the employed systems. The extra regular values can be the results of under over load, over voltage, under or over frequency, and unbalanced operation, e.g., single phasing, etc. One of the key processes of the SCADA systems is the ability to control the devices.

\section{B. Smart Grid.}

The Smart Grid domain is concerned with distributed intelligence such as data decentralization, distributed generation and storage, distribution substation systems and high-voltage transmission networks, distribution system automation and optimization, etc. Involvement and interaction of customer are also considered such as power line-grids and high-consumption electric devices including plug-in hybrid electric vehicles (PHEV).

The Smart Grid is defined as the real-time data and active grid management via fast two-way PLC/optical fiber communications through the applications of technological solutions to the electricity delivery infrastructure. Connectivity exists among and within electric utility, utility's devices, consumer devices, and third-party entities such as vendors, consumers, or regulatory bodies. Smart Grid includes an intelligent monitoring system that tracks the flow of electricity throughout the electrical network, and incorporates the use of superconductive transmission lines to manage power fluctuations, losses, and cogeneration integrations from solar and/or wind.

\section{Smart Grid/SCADA Integration}

SCADA integration into the Smart Grid is not difficult, and connected by both electrical and data networks, allows for central and distributed aggregation of information and control over the entire utility electrical device network.

Digital communications on Smart Grid occur over a variety of devices, technologies, and protocols that include wired and wireless telephones, voice and data dispatch radios, fiber optics, power line carriers, satellites, etc. Decision control software (DCS) allows for dynamic grid management that involves monitoring a significant number of control points. To be fully effective and 
operational, monitoring occurs for every power line and piece of equipment in the distribution system, in addition to allowing the customers to monitor and control their own devices and usage. These result in considerable volumes of data to be organized, analyzed, and used for both manual and automated decision software that comes in two basic categories: decentralized and back office.

\section{III.FRAMEWORK OF POWER LINE /OPTICAL FIBER COMMUNICATION}

\section{A. Framework of the PLC Circuit}

Since the impedance of transmission lines is uniformly distributed, the inductance and capacitance per unit length of transmission lines depend on the employed conductive materials. The function of the PLC signal with respect to the frequency and the distance can be grown into logarithm form.

The coupling unit consists of a capacitor circuit for coupling communication signals, and an inductor circuit for passing low-frequency high power at signal ports $\mathrm{X}$ and $\mathrm{Y}$, and a fusible safety link for protecting the coupling capacitor and inductor from the power line as shown in Fig. 2.

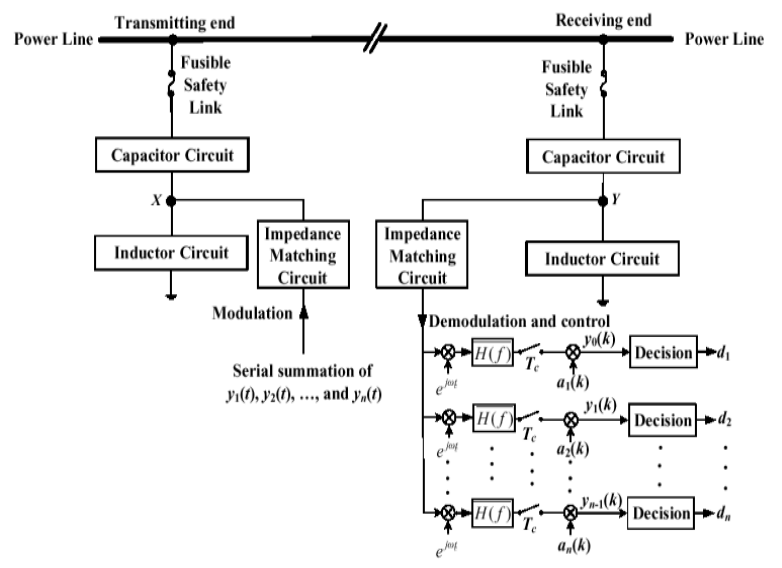

Fig. 2. On-line/download of signals with message on the power line

The serial summation of $\mathrm{y}_{1}(\mathrm{t}), \mathrm{y}_{2}(\mathrm{t}), \ldots, \mathrm{y}_{\mathrm{n}}(\mathrm{t})$ at the transmitted end goes through an impedance-matching circuit to point $X$ and then passes through the capacitor circuit to the power line. The above signals at the receiving end pass through the capacitor circuit to point $Y$ and then pass directly through the impedance-matching circuit to its own receiver port for checking control and turning off the faulted switch shown in Fig. 3.

The signals attenuate with the increase of the transmitted distance in logarithmic form and, hence, they have to be amplified as shown in Fig. 3. The inductor circuit between points $\mathrm{A}$ and $\mathrm{B}$ is for low-frequency signals, and the bidirectional amplifier circuit can be used in bi-directional link port transmission between points $\mathrm{C}$ and $\mathrm{D}$. The signals appearing at point $\mathrm{A}$ go through the capacitor circuit between points $\mathrm{A}$ and $\mathrm{C}$ to the bi-directional amplifier circuit [7]. The amplified signals are divided into two paths: (a) the normal path on which the signals appearing at point $\mathrm{D}$ pass through the capacitor circuit to point $B$, and (b) the abnormal path on which the amplified control signals $\left(d_{0}, d_{1}, \ldots, d_{n-1}\right)$ appearing at point $C$ pass through the capacitor circuit to point $\mathrm{A}$, and then go through the power line to be transmitted.

The signal attenuation depends on the power losses of the path and the variations of the power-line noise. The power losses of the path are dependent on the cable type, cable length, coupling mismatch number, and the number of branches on the power-line network. The path losses increase with the increase of frequency and distance, while the power-line noise decreases with the increase of frequency.

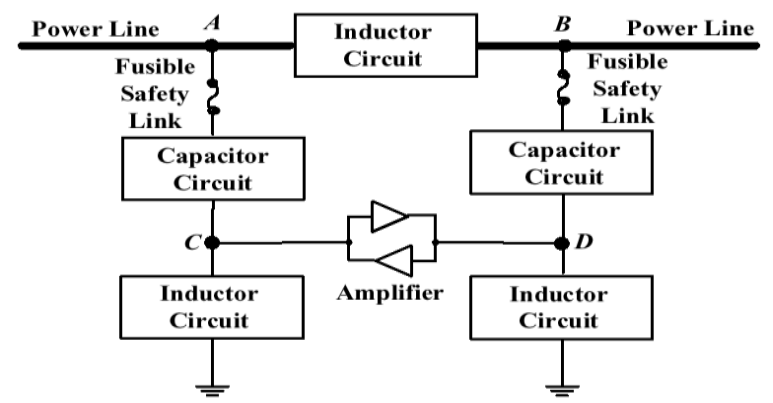

Fig. 3. Signals with data amplified on the power line.

B. Framework of the Electrical Signal/Optical Signal Converter

Fig. 4 shows the electrical/optical signal of a typical receiver and transmitter circuit. In the transmitter side, the electrical signal from the power line is applied to the drive circuit and then to laser diode (LD) which convert the electrical signal to the optical signal. After passing through fiber flylead, the connector, optical fiber, optical splice and optical coupler or beam splitter, the signal is applied to the regenerator and optical amplifier to the receiver side.

On the receive side, the optical signal is applied to a photodetector (PD) which produces a small output current proportional to the optical signal. This current is then amplified and converted to a voltage by a transimpedance amplifier (TIA or TZA). The voltage signal is further amplified by a limiting amplifier (LA) or an automatic gain control (AGC), through the signal restorer the output electrical signal is obtained.

The bit rate of transmitted signal accords with synchronous optical network (SONET)/synchronous digital hierarchy (SDH). American National Standards Institute (ANSI) and International Telecommunication Union (ITU) correspond to different baud rate of SONET/SDH as listed in Table I. The SONET/SDH with respect to the distance can be used for the light source whose operating wavelength ranges from $1330 \mathrm{~nm}$ to 1550 $\mathrm{nm}$ as listed in Table II. 
Table I. Baud rate of transmitted signal with respect to SONET, ANSI and ITU

\begin{tabular}{llll}
\hline SONET & ANSI & ITU & Baud rate (Mb/s) \\
\hline OC-1 & STS-1 & - & 51.84 \\
OC-3 & STS-3 & STM-1 & 155.52 \\
OC-12 & STS-12 & STM-4 & 622.08 \\
OC-24 & STS-24 & STM-8 & 1244.16 \\
OC-48 & STS-48 & STM-16 & 2488.32 \\
OC-96 & STS-96 & STM-32 & 4976.64 \\
OC-192 & STS-192 & STM-64 & 9953.28 \\
\hline
\end{tabular}

Table II. Transmitted distance with respect to different source light for SONET and SDH.

\begin{tabular}{lll}
\hline Transmitted distance & SONET define & SDH define \\
\hline$\leqq 2 \mathrm{~km}$ & Short-reach & Intra-office \\
$15 \mathrm{~km}$ & Intermediate-reach & Short-haul \\
$40 \mathrm{~km}$ at $1330 \mathrm{~nm}$ & Long-reach & Long-haul \\
$80 \mathrm{~km}$ at $1550 \mathrm{~nm}$ & - & - \\
$120 \mathrm{~km}$ at $1550 \mathrm{~nm}$ & - & Very long-haul \\
$160 \mathrm{~km}$ at $1550 \mathrm{~nm}$ & - & Ultra long-haul \\
\hline
\end{tabular}
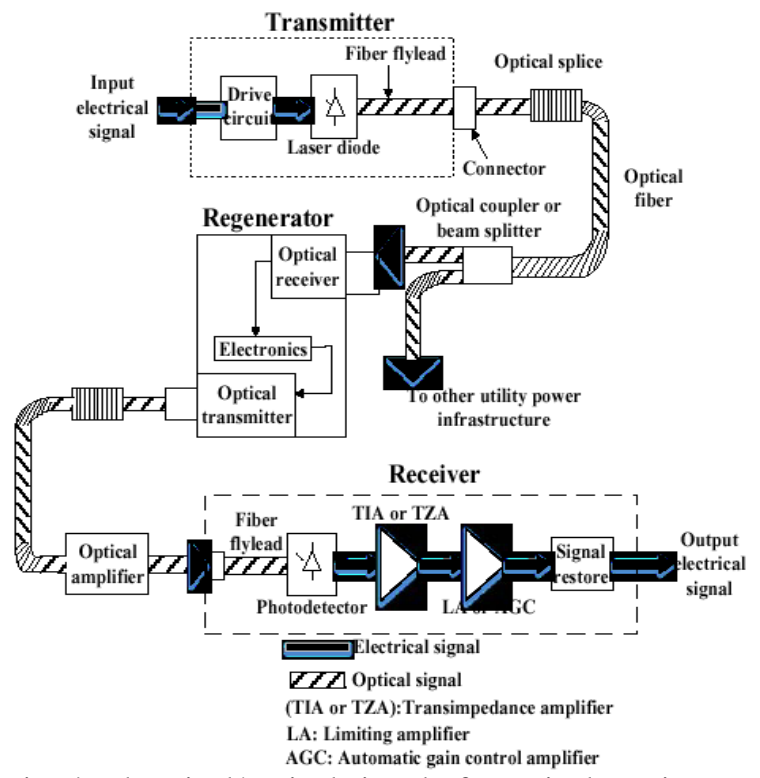

Fig. 4. Electrical/optical signal of a typical receiver and transmitter circuit

\section{Optical Fiber/ Wireless Network}

Fig. 5 shows the architecture model of passive optical network with different optical links. The signal with information makes use of many laser diodes that convert the electrical signal to the optical signal. Optical signals are used by multiplexing through optical fiber links to the receiver side. These are used by demultiplex to several PDs which convert the optical signal to the electrical signal, then after which is used by RoF converter through radio-frequency $(\mathrm{RF})$ wireless transmission, and connects to the supported number of users. Simultaneously, which are transmitted through passive optical networks are sent to central control room in order to monitor and control the SCADA systems.

Wireless and optical communications technologies are combined by the use of two technologies -conventional radio-frequency (RF) wireless and optical fiber for telecommunication. Long distance links are provided by optical fiber from the long distance end points to end consumers, and this RF wireless technology is used for some part of the consumers, as shown in Fig. 5.

The optical/wireless networks offer internet connectivity both to consumers and mobile devices relying on optical, wireless and wired networks for the transport of information. The wireless communication technologies are applied to content distribution, microwave, mobile communication and global positioning for satellite systems.

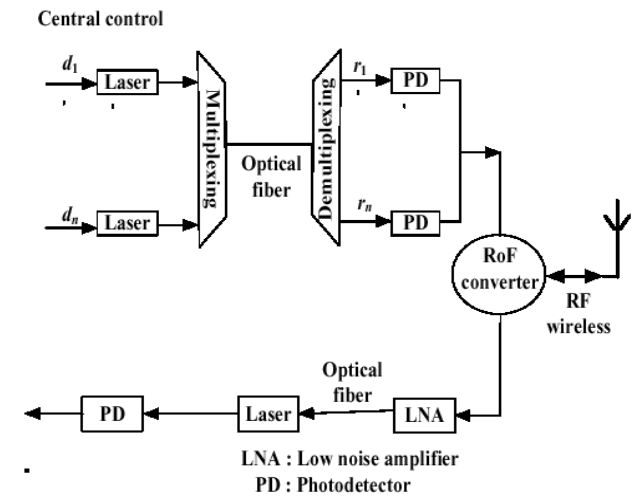

Fig. 5. Simplified optical network architecture model with wire optical links and wireless

D. Framework of the CDMA/ OCDMA and IDMA/ OIDMA

Multiplexing is a way of sending multiple signals or streams of information over a communication link at the same time. The signal with complicated information is spread into many data output lines at the receiver side. Wavelength division multiplexing (WDM) makes the use of division of wavelength with different information to deliver channels at the same time over optical- fiber communication. The multiplexing is divided into time division, frequency division and code-division, such as time-division multiplexing (TDM), frequency-division multiplexing (FDM) and code-division multiplexing (CDM) which are applied to optical communication, which also call sub-carrier modulation (SCM) multiplexing on spectroscope.

In recent years, there have been endeavors to apply multiple access techniques, i.e., code-division multipleaccess (CDMA), interleave-division multiple-access (IDMA) and orthogonal frequency-division multipleaccess (OFDMA) in different systems such as wireless communication system, optical communication system, and power-line communication system (PLCS) Optical CDMA (OCDMA) and optical interleave-division multiple-access (OIDMA) in these endeavors showed good performance in the optical domain [14]. Efficiency, adaptability, safety, security, and reliability are the major features of future electric power systems. The electric power infrastructure requires high spectral efficiency, high 
data rate, high image transmission, high-quality video transmission, low power consumption, low complexity, etc.

Recently, IDMA has been introduced as a new multiple access spread spectrum technique that is similar to CDMA, In IDMA scheme, the user-specific interleaver replaces by using user specific codes as in CDMA scheme. IDMA also allows the use of a low complexity iterative chip-by-chip multi-user detector (MUD) strategy. The computational complexity of the MUD in uplink IDMA systems is a linear function of the number of users, and the MUD in uplink IDMA systems is much simpler than that used in traditional CDMA system.

Performance comparison of IDMA and CDMA has shown that the BER using IDMA performs better than the one using CDMA, IDMA is recently proposed to meet these requirements. Reference introduces OIDMA scheme and offers high bandwidth for higher number of users at minimum cost. The authors in used the on-off-keying (OOK) modulation to simulate an OIDMA scheme over a nonlinear optical channel.

It is demonstrated that OIDMA has better bandwidth utilization and less multiple access interference for the non -coherent intensity modulation/direct detection (IM/DD) optical communication systems, where a unipolar transmission scheme should be used. Similarly, the results of revealed that OIDMA had better BER performance than OCDMA when comparing the performance of both OIDMA and the chip-by-chip decoded OCDMA systems. Therefore, IDMA/CDMA and OIDMA/OCDMA hybrid transmission are applied to electric power system to transmit on either power line communication or fiber communication.

\section{E. CDMA/OCDMA Scheme}

The upper part shown in Fig. 6 illustrates the transmitter with $n$ input data sequence from $n$ users. The $d_{n}=\left[d_{1}(1)\right.$, $\left.\mathrm{d}_{2}(2), \ldots, \mathrm{d}_{\mathrm{n}}(\mathrm{n})\right]$ is encoded by Hadamard code or PN code into $C_{k}=\left[C_{k}(1), C_{k}(2), \ldots, C_{k}(J)\right]$, where $J$ denotes the chip length. After the shaping filter which is modulated with carrier frequency, the spreading signal $\mathrm{y}_{1}(\mathrm{t}), \mathrm{y}_{2}(\mathrm{t}), \ldots$ , $\mathrm{y}_{\mathrm{n}}(\mathrm{t})$ can be obtained. These signals are transmitted through additive white Gaussian noise (AWGN) channel and the transmission power line, which are demodulated with carrier frequency, and after then passing through the shaping filter and PN code to the receiving side. The required data are extracted by decision devices, which are denoted by $r=\left[r_{1}(1), r_{2}(1), \ldots \ldots, r_{n}(n)\right]$.

Similarly, optical code-division multiple access (OCDMA) is an evolution of CDMA with multiuser which are entered each channel at the same time. A CDMA with multiuser system consists of the transmitter side with electrical signal/optical signal converter, the receiver side with optical signal/electrical signal converter, and a spreading operation (each channel for multiuser) [20],

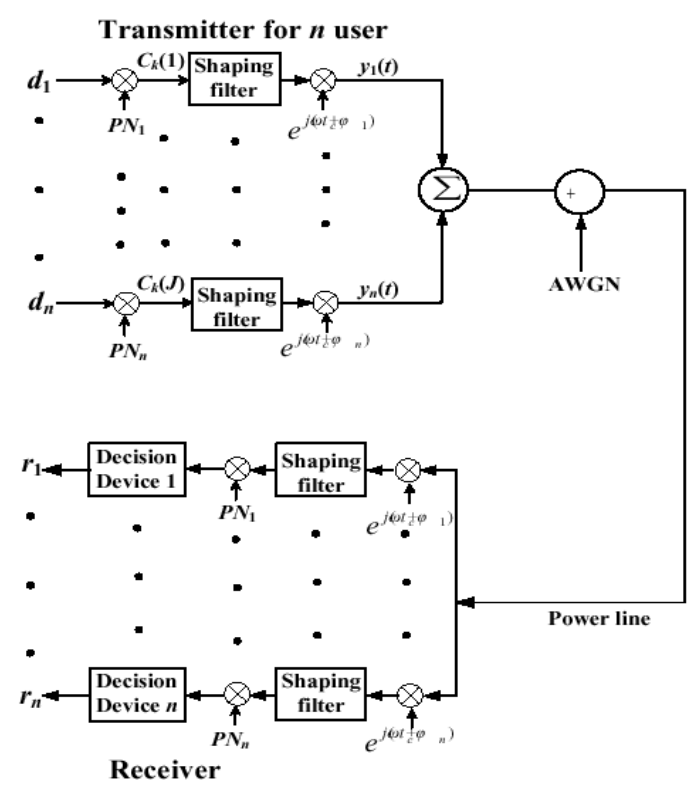

Fig. 6. Transmitter and receiver structures of CDMA scheme with n users.

Similarly, optical code-division multiple access (OCDMA) is an evolution of CDMA with multiuser which are entered each channel at the same time. A CDMA with multiuser system consists of the transmitter side with electrical signal/optical signal converter, the receiver side with optical signal/electrical signal converter, and a spreading operation (each channel for multiuser),

\section{F. IDMA/OIDMA Scheme}

Fig. 7 shows an interleave IDMA system with $\mathrm{K}$ simultaneous users. The upper part shown in Fig. 7 draws the transmitter, where a N-length input data sequence $d_{k}=$ $\left\{d_{k}(j) T, k=1,2, \ldots, N\right\}$ of user-K is encoded by a lowrate code $\mathrm{C}$ (a spreading operation can be merged) into $\mathrm{c}_{\mathrm{k}}$ $=\left\{c_{k}(j) T, j=1,2, \ldots, J\right\}$, where $J$ is the chip length. A spreading operation is following the convention of CDMA.

In encoder -spreader block, the code $\mathrm{C}$ consists of the serial concatenating forward error correction (FEC) code and repletion code of length-sl. The FEC code used in this paper is Memory-2 Rate-1/2 convolutional coder. We may follow the convention of CDMA and call the elements in $c_{k}$ and $x_{k}$ "chip". Then $c_{k}$ are interleaved use of a chip level interleaver ' $\Pi_{k}$ ', generating a transmitted chip sequence $x_{k}=\left\{x_{k}(j) T, j=1,2, \ldots, J\right\}$, then after transmitting through AWGN channel and the transmission power line to the receiving side. The required bits are extracted by signal decoders, which are denoted at the receiver as $r_{k}=\left\{r_{k}(j) T, j=1,2, \ldots, J\right\}$.

Similarly, optical interleave-division multiple access (OIDMA) is an evolution of OCDMA with FEC. An OCDMA with FEC system consists of the concatenation of a FEC code, a spreading operation (unique for each user), and an interleaver (common to all users), 
Transmitter for user- $k$

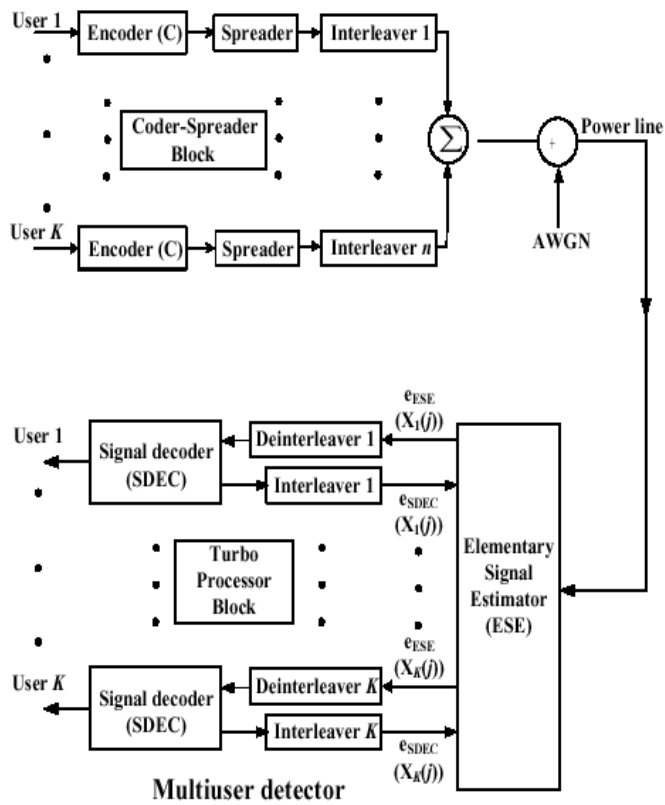

Fig. 7. Transmitter and receiver structures of an IDMA with $\mathrm{K}$ simultaneous users

\section{IV.CONCLUSION}

Implementation of optical fiber technology provides many advantages over wired or wireless, e.g, low installation cost, rapid bit rate. Wide band width, less BER, Longer transmitted distance. Reduced interference among them, lower signal attenuation, higher capacity, etc. However, each technology has certain Challenges, which need to be addressed for its future use in smart Grid environment. Few Common concerns for power line/optical fiber technologies are listed as below.

[1] Both IDMA/CDMA and OIDMA/OCDMA hybrid transmission architecture employ OIDMA/OCDMA technique as the uplink transmission and CDMA/OCDMA technique as the downlink transmission for monitoring and control.

[2] Optical fiber technologies offer wide bandwidth to multiple users, depending on tolerability within operating bandwidth, that enter each channel at the same time in order to increase transmitted capacity;

[3] Cross-talk and mutual interference are not produced among optical fiber communications so as to achieve security and reliability.

\section{REFERENCES}

[1] Chuang and M. McGranaghan, "Functions of a local controller to coordinate distributed resources in a smart grid," in Proc. IEEE PESGen. Meeting, July 2008, pp. 1-6.

[2] T. F. Garrity, "Getting smart," IEEE Power Energy Magazine, vol. 6, no. 2, pp. 38-45, Mar./Apr. 2008.

[3] E. Lambert, J. Fremont, and C. Bouquet, "Method and applications of IEC common information model standard for distribution operations: A path towards smart grids development," in Proc. IETCIRED Seminar SmartGrids Distrib., Jun. 23-24, 2008, pp. 1-4.

[4] M. H. Oxrnan, Bi-directional Signal Circuit, Patent US3175050, 1965.

[5] J. Gowar, Optical Communication Systems, New York: Prentice Hall, 1993.

[6] G. Keiser, Optical Fiber Communications, New York: McGrawHill, 2000.ANSI T1.105.06 Specification. ITU-T G.957 Specification.

[7] Cox, E. Ackerman, R. Helkey, and G. E. Betts, "Direct detection analog optical links," IEEE Trans. Microwave Theory and Techniques, vol. 45, no. 8, pp. 1375-1383, Aug. 1997.

[8] J. Bowers, A. Chipaloski, S. Boodaghians, and J. Carlin, "Long distance fiber-optic transmission of C-band microwave signals to and from a satellite antenna," Journal of Lightwave Technology, vol. 5, no. 12, pp. 1733-1741, Dec. 1987.

[9] J. A. Salehi, "Code division multiple access techniques in optical fiber networksart, I: Fundamental principles," IEEE Trans. Communication, vol. 37, pp. 82433, Aug. 1989.

[10] J. Armstrong, "OFDM for optical communications," J. Lightwave Technology, vol. 27, no. 3, pp. 2792-2799, July 2009.

[11] L. Ping, L. Liu, K. Y. Wu, and W. K. Leung, "Interleave-division multipleaccess (IDMA) communications," in Proc. 3rd International Symposium on Turbo Codes and Related Topics, Sep. 2003, pp. 173-180.

[12] L. Ping, L. Liu, K. Y. Wu, and W. K. Leung, "Interleave-division multiple access," IEEE Trans. Wireless Communications, vol. 5, no. 4, pp. 938-947, Apr. 2006

[13] L. Liu, W. K. Leung, and L. Ping, "Simple chip-by-chip multi-user detection for CDMA systems," in Proc. IEEE VTC003-Spring, Jeju, Korea, Apr. 2003, pp. 2157161.

[14] Bmou and A. Glavieux. "Near Shannon limit error correcting coding and decoding: Turbo codes," IEEE Trans. Communication, vol. 44, no. 10, pp. 1261-1271, Oct. 1996

[15] X. Wang and H. V. Poor, "Iterative (turbo) soft interference cancellation and decoding for coded CDMA." IEEE Trans. Communication, vol. 47, no. 7, pp. 1046-1061, Jul. 1999.

[16] R. H. Mahadevappa and J. G. Raakis, "Mitigating multiple access interference and intersymbol interference in uncoded CDMA systems with chip-level interleaving," IEEE Trans Wireless Communication, vol. 1, no. 4, pp. 781-792, Oct. 2002.

[17] W. K. Leung, L. Liu, and L. Ping, "Interleaving-based multiple access and iterative chip-by-chip multiuser detection," IEICE Trans. Communication, vol. E86-B, no. 12, pp. 3634-3637, Dec. 2003.

[18] X. Liu, X. Xiong, and P. Yang, "Performance of IDMA and CDMA systems based on Simulink," Wireless Communications Networking and Mobile Computing (WiCOM), Sep. 2010, pp. 1-4.

[19] M. Shukla1, M. Gupta, S. Tiwari, P. S. Sharma, and S. Shukla, "Optical interleave-division multiple-access scheme for long distance optical fiber communication," 2010 IEEE International Conference on Computational Intelligence and Computing Research, Dec. 2010, pp. 260-263.

[20] M. Morsy, E. A. El-fiky, H. S. Khallaf, and H. M. H. Shalaby, "Performance analysis of optical IDMA and optical CDMA techniques using unipolar transmission scheme," in Proc. 18th European Conference on Network and Optical Communications \& 8th Conference on Optical Cabling and Infrastructure, Jul. 2013, pp. 55-59.

[21] K. Kitayama, "Novel spatial spread spectrum based fiber optic CDMA networks for image transmission," IEEE Journal on Selected Areas in Communications, vol. 12, no. 4, pp. 762-772, May 1994. 